\title{
Identification of Efficient Sampling Techniques for Probabilistic Voltage Stability Analysis of Renewable-Rich Power Systems
}

\author{
Mohammed Alzubaidi ${ }^{1,2, *}$, Kazi N. Hasan ${ }^{1}\left[\right.$, Lasantha Meegahapola ${ }^{1}\left(\mathbb{D}\right.$ and Mir Toufikur Rahman ${ }^{1}$ \\ 1 Electrical and Biomedical Engineering, School of Engineering, RMIT University, Melbourne 3001, Australia; \\ kazi.hasan@rmit.edu.au (K.N.H.); lasantha.meegahapola@rmit.edu.au (L.M.); \\ toufik.rahman@rmit.edu.au (M.T.R.) \\ 2 College of Engineering, Umm Al-Qura University, Al-Qunfudhah 21912, Saudi Arabia \\ * Correspondence: s3583733@student.rmit.edu.au
}

Citation: Alzubaidi, M.; Hasan, K.N.; Meegahapola, L.; Rahman, M.T. Identification of Efficient Sampling Techniques for Probabilistic Voltage Stability Analysis of Renewable-Rich Power Systems. Energies 2021, 14 2328. https://doi.org/10.3390/ en14082328

Academic Editor: Adrian Ilinca

Received: 31 March 2021

Accepted: 17 April 2021

Published: 20 April 2021

Publisher's Note: MDPI stays neutral with regard to jurisdictional claims in published maps and institutional affiliations.

Copyright: (c) 2021 by the authors. Licensee MDPI, Basel, Switzerland. This article is an open access article distributed under the terms and conditions of the Creative Commons Attribution (CC BY) license (https:// creativecommons.org/licenses/by/ $4.0 /)$.

\begin{abstract}
This paper presents a comparative analysis of six sampling techniques to identify an efficient and accurate sampling technique to be applied to probabilistic voltage stability assessment in large-scale power systems. In this study, six different sampling techniques are investigated and compared to each other in terms of their accuracy and efficiency, including Monte Carlo (MC), three versions of Quasi-Monte Carlo (QMC), i.e., Sobol, Halton, and Latin Hypercube, Markov Chain MC (MCMC), and importance sampling (IS) technique, to evaluate their suitability for application with probabilistic voltage stability analysis in large-scale uncertain power systems. The coefficient of determination $\left(\mathrm{R}^{2}\right)$ and root mean square error (RMSE) are calculated to measure the accuracy and the efficiency of the sampling techniques compared to each other. All the six sampling techniques provide more than $99 \%$ accuracy by producing a large number of wind speed random samples (8760 samples). In terms of efficiency, on the other hand, the three versions of QMC are the most efficient sampling techniques, providing more than $96 \%$ accuracy with only a small number of generated samples (150 samples) compared to other techniques.
\end{abstract}

Keywords: probabilistic techniques; uncertainty modelling; voltage stability; wind power generation

\section{Introduction}

Power system stability has gained more attention in modern power system analysis due to the increased penetration of intermittent renewable energy sources (RES) and the operation of the electricity markets. For the economic operation of the power systems, the network has been operated close to its voltage stability boundary [1,2]. This has arisen from the growing usage of distributed generation of RES, and the intrinsic heterogeneity of grid loads, which increases the number of uncertainties in power system networks [3]. The intermittency of the RES coupled with the variability of system loads can increase the risk of voltage instability. The variability presented by the RES and system loads is not considered in the traditional deterministic stability analysis, where the worst-case scenario is usually considered for system behavior prediction $[4,5]$. Therefore, probabilistic voltage stability analysis considering a wide range of the variability of the system parameters is imperative in power system planning and operation [6]. Hence, the probabilistic method can accurately provide a better reflection of the actual system behavior by explicitly taking into account the uncertainties of the system parameters and stochastic variability in parameters, disturbances, and operating conditions $[7,8]$.

The probabilistic technique aims to develop the operational uncertainties of a system in order to demonstrate the system behavior over a wide range of possible scenarios. The techniques generally emphasis the computational efficiency and accuracy of the desired uncertain variables. In the literature, various probabilistic techniques have been utilized for probabilistic voltage stability analysis, including Monte Carlo (MC) simulation [2,9-12], 
Quasi-Monte Carlo (QMC) [13,14], maximum entropy method [1,15], probabilistic collocation method [16,17], and cumulants method $[1,18]$. Apart from these, the point estimate method [19] and sequential MC method [20] have also been used in power system studies.

Although MC is the most widely used benchmark method for probabilistic simulation, it requires a higher number of simulations and computational time to obtain the actual system response with various uncertainties in the power system network [21,22]. Thus, MC is not considered efficient in large systems with many uncertain parameters, particularly for high-resolution dynamic studies [21,22]. In order to overcome the drawbacks of MC, various probabilistic techniques have been independently applied. For instance, QMC is used to model the wind power uncertainties [13] and electric vehicle characteristics [14] for voltage stability analysis. The maximum entropy method is used to represent the wind power uncertainties [1] and load variations [15] in large-scale power systems. Furthermore, the probabilistic collocation method [17] and the cumulant method [1] are also used to model the wind power uncertainties for voltage stability analysis. On the other hand, in the absence of any renewable generation into the network, probabilistic techniques are used to model the load variations [18] and uncertainties in the power oscillation damping controller [16]. However, these studies do not comparatively measure the efficiency and accuracy of probabilistic methods. Thus, it is essential to identify efficient sampling techniques to accurately assess the influence of the uncertainties in the network and their impact on probabilistic voltage stability analysis.

This paper, for the first time, presents a comparative analysis of probabilistic techniques to identify an efficient and accurate sampling technique to be applied to probabilistic voltage stability analysis in large-scale power systems. The intermittency of the wind generation and uncertainties in system loads over a year are considered as input variables of the sampling techniques. Six widely used probabilistic techniques, including $\mathrm{MC}$, three variants of QMC, MCMC, and importance sampling (IS) technique, are compared in terms of their efficiency and accuracy. The IEEE-39 bus network connected with three wind farms is utilized to demonstrate the most efficient and accurate probabilistic technique for the voltage stability analysis. The PV-curve analysis is conducted in DIgSILENT PowerFactory software to determine the loadability of the system. The coefficient of determination $\left(R^{2}\right)$ and root mean square error (RMSE) are calculated to measure the accuracy of the sampling techniques.

The main contribution of this paper can be highlighted as three key points, which include:

- Implementing six sampling techniques with significantly various computational principles to generate accurate uncertainties of wind speed in the sample dataset.

- Evaluating the accuracy (coefficient of determination $\left(R^{2}\right)$ and root mean square error (RMSE)) and efficiency (number of simulation) of applied sampling techniques for modelling uncertainties of wind speed sample data generation.

- Identifying the most accurate and efficient sampling technique in order to probabilistically analyze the voltage stability, considering a wide range of uncertainties in wind speed sample dataset to be applied in large-scale power systems.

The rest of the paper is organized as follows. Section 2 delineates a theoretical analysis of the probabilistic voltage stability and the probabilistic techniques; Section 3 demonstrates the uncertainty modeling and the research methodology, followed by simulation results and discussion in Section 4, and finally, conclusions will be presented in Section 5.

\section{Theoretical Analysis of Probabilistic Voltage Stability and Efficient Sampling Techniques}

2.1. Voltage Stability

Voltage stability is an essential aspect of power system stability, and it will be significantly affected by the natural variability of wind power generation. Voltage stability refers to the ability of system voltages to recover and maintain the acceptable voltage range following a contingency in the power grid [23]. During a voltage instability event 
driven by a small perturbation, voltage declines progressively in a busbar, in a small area of a network, or the whole network, and then it quickly decreases and subsequently collapses [24]. Consequently, the power system operations are significantly affected. Voltage stability issues are typically observed in highly loaded power networks. A variety of causes contribute to the voltage stability issues, such as reactive power deficit due to the limitations in generators while increasing the load demand $[25,26]$, and malfunction in voltage control devices [26]. The P-V curve method is an accurate and most widely used method in assessing voltage stability. The loadability (or load margin, which will be used in this paper interchangeably), is considered as the voltage stability index [27]. The loadability presents the maximum active power capability prior to the voltage collapse [23].

\subsection{Probabilistic Voltage Stability}

The probabilistic method can present a much more realistic view of the expected system behavior and a more reliable and accurate prediction of the static and dynamic system response $[7,8]$. Figure $1 \mathrm{a}, \mathrm{b}$ shows the P-V curve and the probabilistic loadability of the critical busbar for 8760 simulations (representing hours of the year) by considering the wind speed and system load uncertainty. It can be observed from Figure 1a that the knee point of the P-V curve can be strongly affected ( $6500 \mathrm{MW}$ to $7800 \mathrm{MW})$ by the variation of the wind speed (from 0 to $14 \mathrm{~ms}^{-1}$ ) and the load of the system (5\% variation with respect to the mean value), which influences the loadability of the power network. These results clearly show the importance of considering the uncertainties in the power network and their impact on voltage stability.

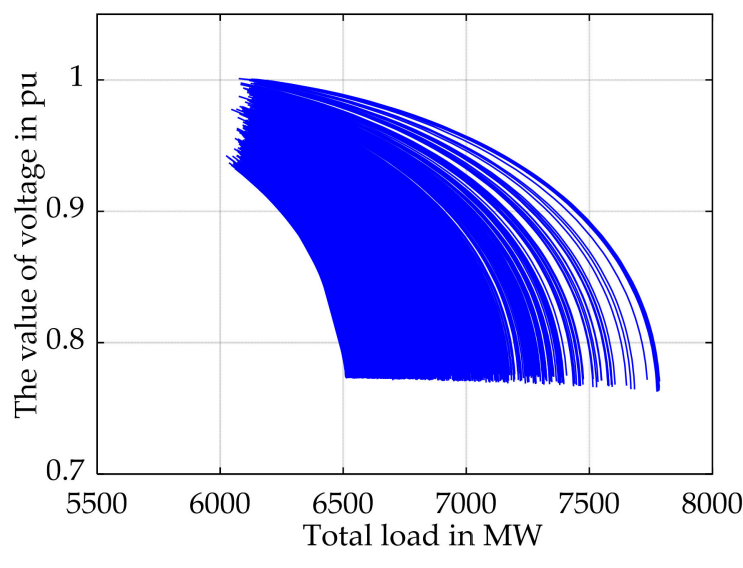

(a)

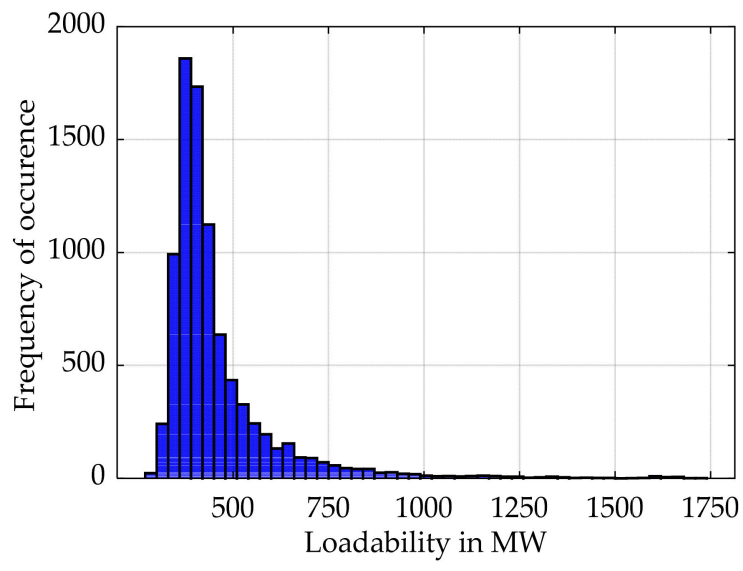

(b)

Figure 1. Probabilistic voltage stability results obtained from 8760 Monte Carlo simulations by considering the uncertainties in the power network: (a) the P-V curve; (b) histogram of the system loadability.

\subsection{Probabilistic Sampling Techniques \\ 2.3.1. Monte Carlo (MC) Simulation}

The MC is the most widely used method for probabilistic simulations. This simulation model works by compiling a large data set of uncertain parameters from a given system. This dataset is built by sampling variables and provides the probability distribution of an unknown variable, and this function is known as an output probability density function. This type of simulation defines the possible input parameters for a given system, sample random data out of those inputs, and process them in order to provide output results. As the whole method depends on the sampled data, the accuracy of this simulation increases with an increase in the number of samples and simulations [28,29]. The MC method has been applied in different aspects of power systems stability applications, such as voltage stability $[9,10]$, frequency stability [30,31], transient stability [32,33], and small-disturbance stability [19]. 
The MC method, since it is based on a numerical solution, requires a large number of repeated samples to accurately represent the stochastic behavior of uncertain system variables. To determine a sufficient number of the MC in order to obtain the accurate result, the stopping rule is applied as described in the following equation [34].

$$
\varepsilon=\left[\frac{\varnothing^{-1}\left(1-\frac{\delta}{2}\right) \cdot \sqrt{\frac{\alpha^{2}(x)}{N}}}{\bar{X}}\right]
$$

In [1], $\varepsilon$ is considered to be the sample mean error, the $\varnothing^{-1}$ is the standard inverse Normal probability distribution with a zero mean and one standard deviation value. $\alpha^{2}(x)$ is the variance of the random samples, $\bar{X}$ is the mean of the random samples, and $\delta$ is the required confidence interval.

The calculated error of voltage stability vs. the number of MC simulations is shown in Figure 2 (by considering 95\% and 99\% confidence intervals). It can be seen clearly from Figure 2 that the sample mean error of the load margin is decreased by increasing the number of simulations. Accordingly, to obtain an acceptable result with less than $2 \%$ error for both intervals, 2000 simulations must be performed for the MC method. This means that by running a couple of thousands of MC simulations, an accurate result can be obtained. However, running a smaller number of MC simulations (i.e., a few hundred) may not provide an accurate and acceptable result. Hence, if the number of simulations is required to be low for a large power system, the MC method may not be appropriate, and some other more efficient methods may provide better results.

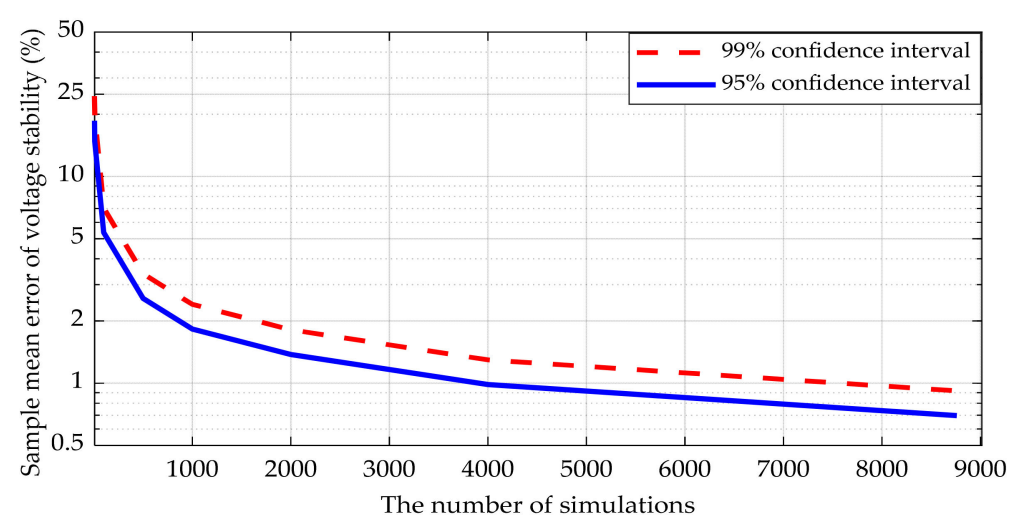

Figure 2. The accuracy vs. efficiency curve of the voltage stability shows the sample mean error of voltage stability against the number of simulations for $95 \%$ and $99 \%$ confidence intervals.

\subsubsection{Quasi-Monte Carlo (QMC) Method}

The basic principle of this method is very similar to the standard MC. The differences occur in the creating of data samples. The standard MC method samples the variables or parameters which are equally probable while QMC samples equidistant samples. The QMC involves the usage of low discrepancy sequences, such as Sobol, Halton, and Latin Hypercube sequences. The basic simulation requirements involve a description of the system inputs in terms of expectation, standard deviation, third and fourth moments, correlation matrix, and the length of the desired low-discrepancy sequences. Based on the above-mentioned parameters, the simulation generates a uniform distribution sequence and performs analysis on variables obtaining numerical results for the uncertainty of distributions $[35,36]$. The QMC approach has been employed in different power systems studies, including small-disturbance stability [35] and probabilistic optimal power flow [36].

In this study, Sobol, Halton, and Latin Hypercube sampling techniques have been implemented. The 150, 300, 1000, and 8760 generated random samples of the MC method (pseudorandom), and QMC method (Sobol, Halton, and Latin hypercube) are shown in Figure 3. Figure 3 can clearly show the difference between the random samples generated 
by the MC and QMC, which indicates that the QMC random samples are based on equally distributed sequences and have more uniform behavior, whereas the MC produces random number samples based on nonuniformly distributed behavior throughout the whole space. It is evident from Figure 3 that when a large number of samples (such as 1000 to 8760) are generated both MC and QMC techniques cover the full searching space, but for a small number of samples, QMC (i.e., Sobol and Halton) coverage of the searching space is more uniform and may provide better results.
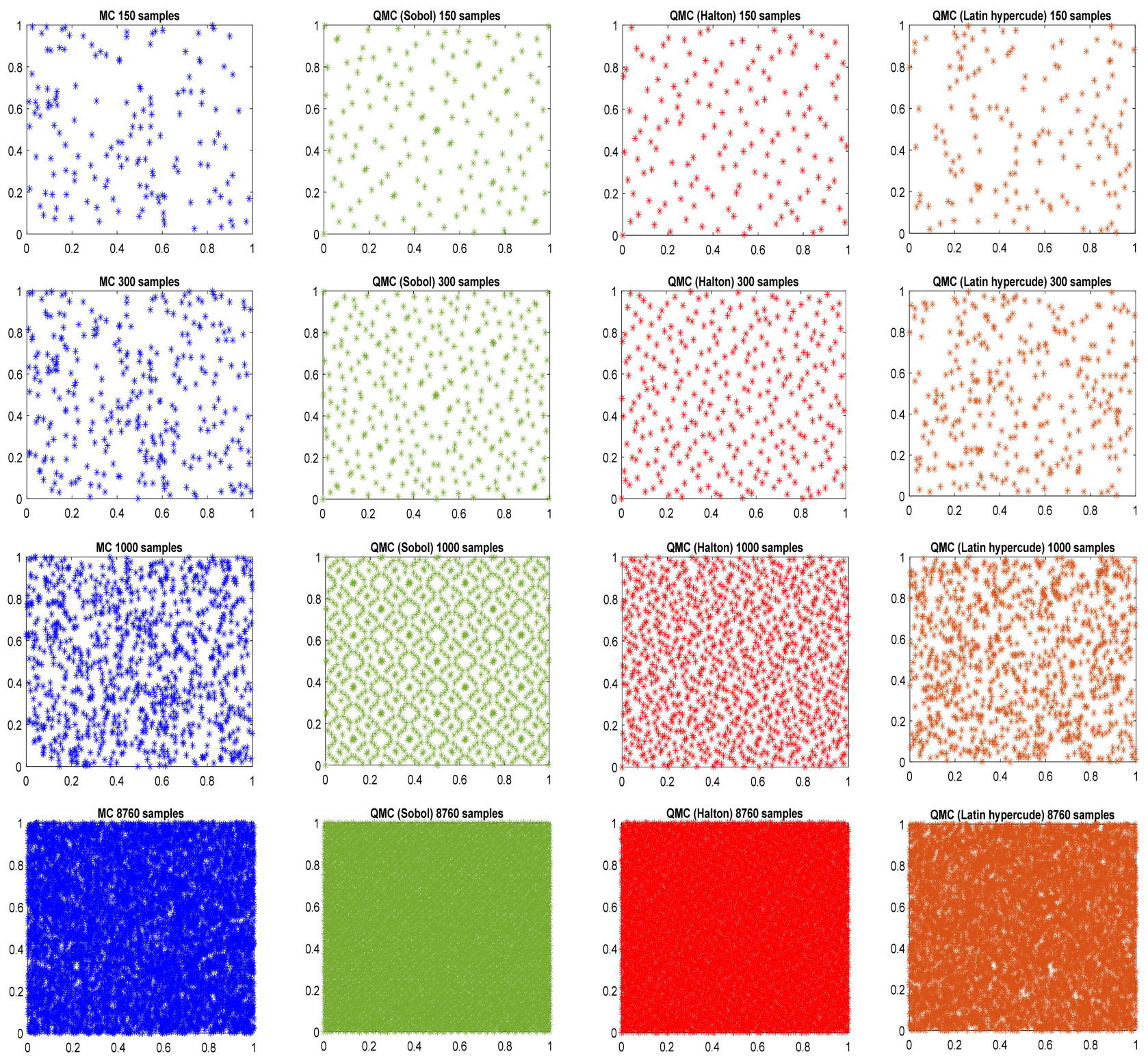

Figure 3. 150, 300, 1000, and 8760 random samples of MC method and three versions of QMC method (Sobol, Halton, and Latin hypercube).

\subsubsection{Marcov Chain MC (MCMC) Method}

The MCMC method is typically employed when a target distribution is difficult to sample directly. To compensate for this, the MCMC takes samples from the probability distribution by constructing a Markov Chain with the target distribution, which can be used to sample the desired distribution. The accuracy of this method also increases with an increase in the size of samples taken at each step. The rest of the procedure for this type of simulation is very similar to the MC, which is applying the deterministic approach to the data and then analyzing it. However, the MCMC improves its performance by eliminating 
the sample generation step of the standard MC method. The main steps to generate random samples by using the MCMC are as follows: initialization of random starting sample, generation of points based on the adopted distribution, repetition of sampling until convergence, and obtaining desired distributions and target variables [7,37]. In this study, the slice sampling technique is used to produce the MCMC sample dataset [38]. The MCMC method has been used in different power systems studies, including voltage stability enhancement [39] and transient stability evaluation [40].

\subsubsection{Importance Sampling (IS) Technique}

The IS technique can be considered as the MC method in which a proposal distribution is employed in order to calculate the importance weight of the distribution. This can assist in resampling the generated random samples based on their importance weight. By considering the $f(x)$ as the target distribution, $g(x)$ as the proposal distribution, and $w(x)$ as the importance weight, the IS sample can be generated by the following steps [41]: (1) drawing random samples from the proposal distribution $(g(x))$, (2) determining the size of the samples and then calculating their probability for each sample, (3) calculating the importance weights, which is equal to $f(x) / g(x)$, and (4) resampling the dataset based on the importance weight. The IS technique has been applied in various power systems studies, including small-disturbance stability [21], power system security [42], and reliability [43].

Some other sampling techniques have not been considered in this study, such as spatiotemporal kriging and analog approaches [44], polynomial chaos expansion [45,46], graphbased convolution networks [47], multi-channel ARMA models [48], dynamic uncertainty sets [49], and universal kriging models [50]. These sampling techniques are extensive in implementation complexity and computational cost and remain an ongoing area of research concerning their appropriateness for application in probabilistic voltage stability analysis by explicitly taking into account the inherent variability of system loads and the intermittent nature of the RESs.

\subsection{Goodness-of-Fit Measures}

Various goodness-of-fit measures can be applied to evaluate the accuracy of the selected appropriate sampling techniques. In this study, the $\mathrm{R}^{2}$ and RMSE measures are implemented to identify the most efficient sampling technique to be used for probabilistic voltage stability analysis. The $\mathrm{R}^{2}$ and RMSE values can be calculated by using (2) and (3), respectively [51].

$$
\begin{gathered}
\mathrm{R}^{2}=1-\frac{\sum_{i=1}^{n}(x i-y i)^{2}}{\sum_{i=1}^{n}(x i-z i)^{2}} \\
\mathrm{RMSE}=\sqrt{\frac{\sum_{i=1}^{n}(x i-y i)^{2}}{n}} \\
z i=\frac{1}{n} \sum_{i=1}^{n} x i
\end{gathered}
$$

In (2) and (3), $i$ is the index value, where $i$ equals $1,2,3, \ldots, n$, and $n$ is the size of the dataset, $x i$, and $y i$ are the reference data and generated samples, respectively. The $z i$ is the mean value of reference data, which can be calculated as described in (4).

\section{Research Methodology and Uncertain Modelling}

\subsection{Research Methodology}

The proposed methodology of this research is shown in Figure 4, which can be divided into two separate sections, which involve: (1) generating wind speed random samples from the six sampling techniques, and (2) analyzing the system loadability by performing continuation power-flow for the reference sample and the generated samples that follow the six sampling techniques. To achieve the main objective, the MC method is applied to generate 8760 samples, which are considered to be the reference dataset, to represent 
the actual wind speed data. Various sampling techniques are implemented to generate random samples with different sizes to be compared with the reference data to identify an accurate and efficient sampling technique to be used for probabilistic voltage stability analysis. These sampling techniques include MC, three versions of QMC, Markov Chain MC (MCMC), and Importance Sampling (IS) technique. The generated samples are used to perform the continuation load flow to plot the P-V curve for the critical busbar, and then, the PDF (probability density function) of the system loadability for the reference samples and the generated samples with a different number of samples are plotted. Finally, based on the RMSE and $R^{2}$ values of the generated samples, and the system loadability, the most accurate and efficient sampling techniques are identified.

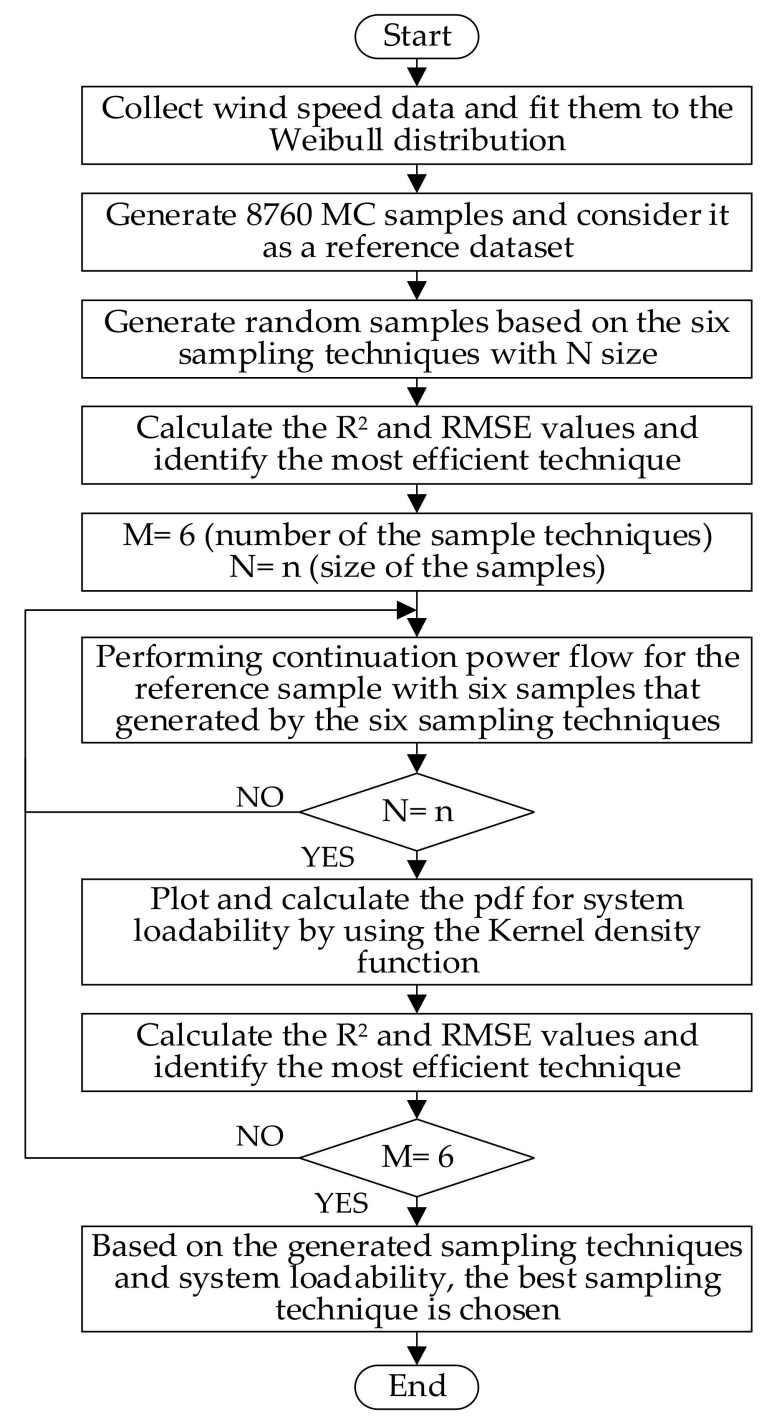

Figure 4. The flowchart of the proposed research methodology of this study.

\subsection{System Description}

A modified version of the IEEE-39 bus network with three wind farms is used in this study for probabilistic voltage stability simulation (data of this network can be found in [52]), which is presented in Figure 5. The integration of the RES is simulated by using three wind farms, which are connected to the system busbars 30, 34, and 37. Three synchronous generators of this network are replaced by wind farms, and the total contribution of the wind farms is considered to be approximately $20 \%$ of the total generation capacity of the system. In this study, two major software platforms, which include MATLAB and DIgSILENT PowerFactory, are employed to perform the probabilistic voltage stability analysis. 


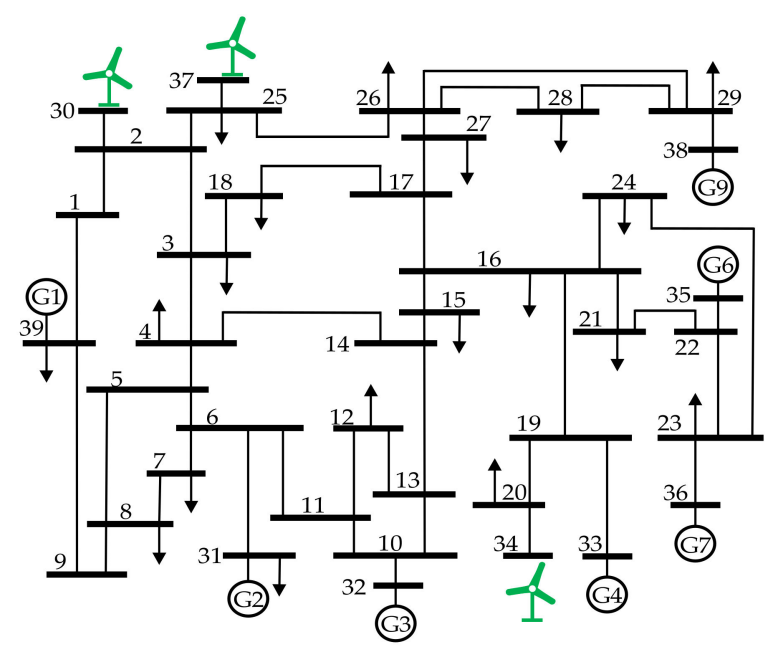

Figure 5. The IEEE-39 bus network integrated with three wind farms.

\subsection{Uncertainty Modelling}

\subsubsection{System Loads}

Power system loads are changing continuously, and they are modelled as an uncertain parameter. The Normal distribution is typically applied to represent the uncertainty of loads. Consequently, it is used in this study to model the variation of the system loads, which is considered to be $5 \%$ of the mean of the Normal distribution. This variation describes the system load forecasting error that happens over $24 \mathrm{~h} \mathrm{[34].}$

\subsubsection{Wind Farms}

In this research, the hourly wind speed dataset for one year is collected from Northolt in the UK [53]. The wind farms, located at system busbars 30, 34, and 37, are fed with the wind speed datasets modelled by using the Weibull distribution, which is commonly used in the literature to represent the wind speed variation [2,54]. The Weibull distribution is presented in (5), and the scale and shape parameters are $k=4.31$ and $c=1.84$, respectively.

$$
f(x ; k, c)=\frac{k}{c}\left(\frac{x}{c}\right)^{k-1} e^{-\left(\frac{x}{c}\right)^{k}}
$$

\section{Simulation Results and Discussion}

4.1. Generating Samples from Different Sampling Techniques and Comparing them with the Reference Dataset

The accuracy of the MC method is increased by increasing the number of simulations, as shown in Figure 2. Therefore, the reference dataset is considered to be generated by the MC method with 8760 samples. This large number of simulations provides an accurate result to be compared with the six sampling techniques that are employed to generate wind speed dataset with different sizes in order to identify the most efficient and accurate sampling technique. Figure 6 presents the actual wind speed data fitted to the Weibull distribution. It can be observed from Figure 6 that the Weibull distribution provides a good representation for the actual wind speed data.

Moreover, the PDF of the wind speed datasets generated by the six sampling techniques with different sizes, including 150,300,1000, and 8760, compared to the reference data (represented by the solid blue line) is shown in Figure 7. In terms of accuracy, it can be seen from Figure 7 that the accuracy of each sampling technique is increased, and particularly rapidly increased in the MC and MCMC methods, by increasing the number of the generated samples to represent the reference dataset. This observation is expected since increasing the number of the generated samples can lead to covering the entire space, especially for the MC, as presented in Figure 3. This result can be clearly observed in Figure $7 \mathrm{~d}$, which shows that all the sampling techniques ideally represent the reference 
dataset. This result has been validated by calculating the RMSE and $R^{2}$ values, which show that the RMSE values are decreased, and the $\mathrm{R}^{2}$ values are increased for each technique by increasing the number of sample generations, as shown in Figure 8.

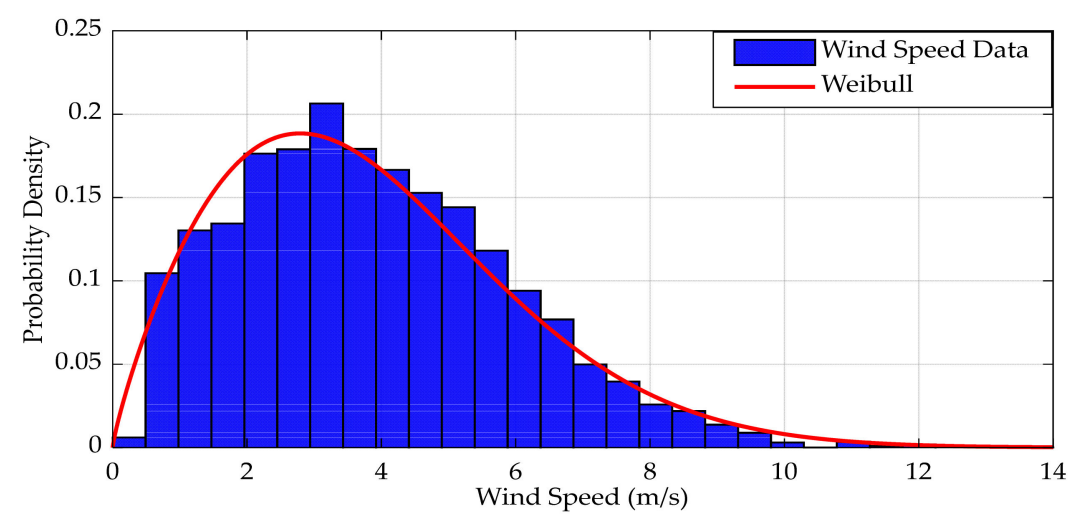

Figure 6. The Actual wind speed data fitted to the Weibull distribution.

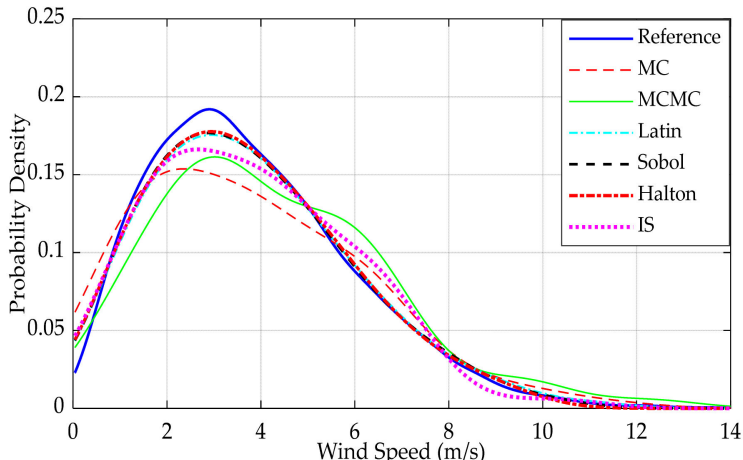

(a)

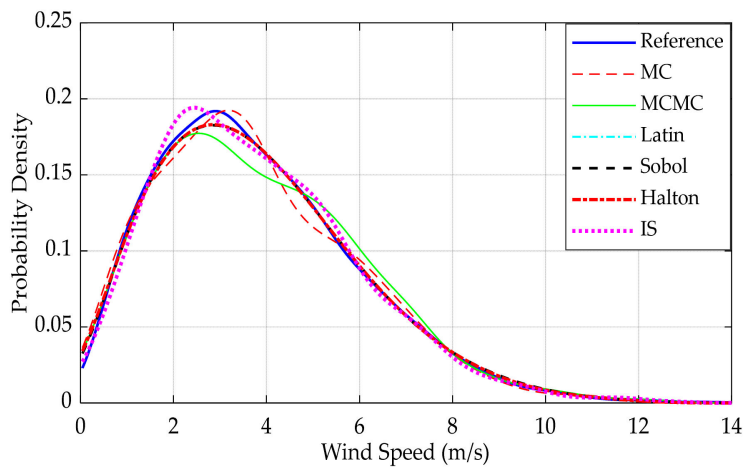

(c)

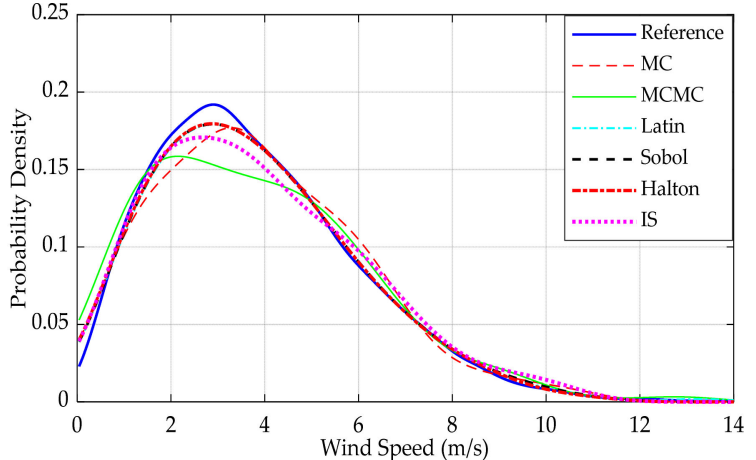

(b)

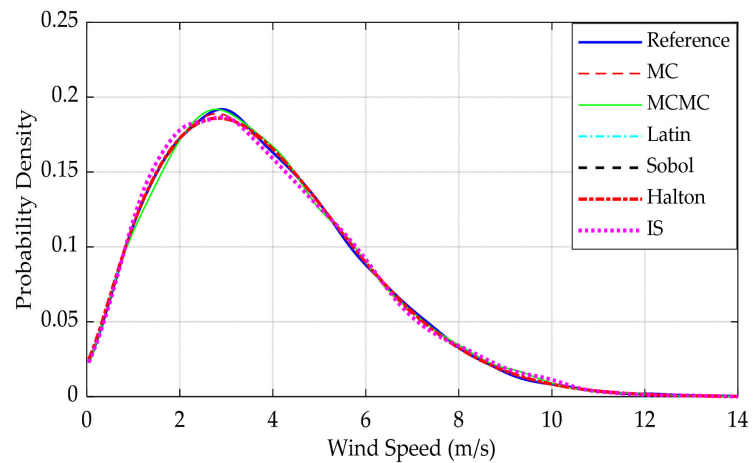

(d)

Figure 7. The PDF of the wind speed datasets generated by the six sampling techniques compared to the reference data: (a) 150 samples; (b) 300 samples; (c) 1000 samples; (d) 8760 samples (cover the number of hours of the year).

In terms of efficiency, on the other hand, it can be observed from Figure $7 \mathrm{a}, \mathrm{b}$ that the accuracy of sampling techniques (by considering fewer samples 150, 300) is different from one technique to another. The three versions of the QMC method, including Sobol, Halton, and Latin hypercube, are the best efficient sampling techniques representing the reference data compared to the other techniques. This result is also confirmed with the RMSE and $\mathrm{R}^{2}$ values, as presented in Figure 8. The lowest value of the RMSE and the highest value of the $\mathrm{R}^{2}$ will assist to determine the most efficient and accurate sampling technique. This observation is reasonable since the three versions of the QMC method are produced lowdiscrepancy samples, which are distributed uniformly to cover the entire space with only 
fewer samples in comparison to other sampling techniques, such as the MC method, which produces random number samples throughout the entire space nonuniformly (as shown in Figure 3). This is why the MC method cannot be considered as an efficient method, and therefore, it is practically not suitable to be utilized in reality for a lower number of simulations.

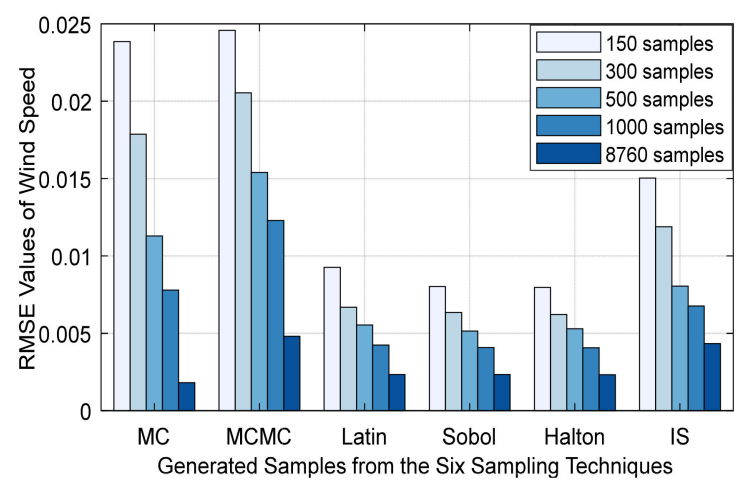

(a)

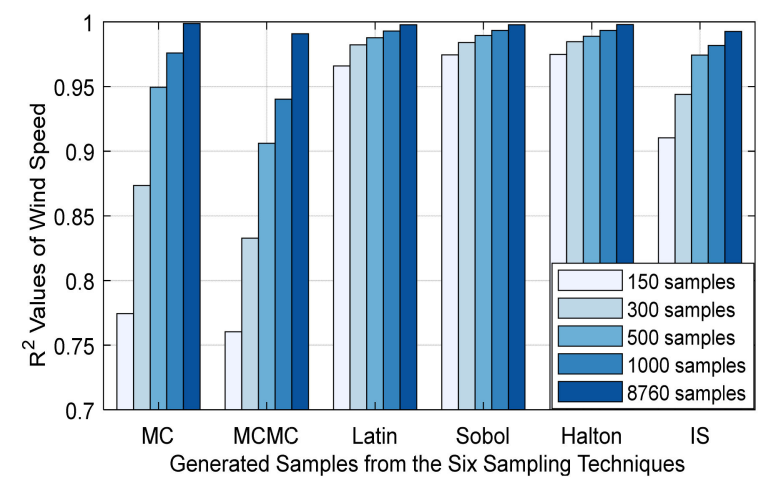

(b)

Figure 8. The (a) RMSE and (b) $\mathrm{R}^{2}$ values of 150, 300, 500, 1000, and 8760 generated samples from the six sampling techniques compared to the reference dataset. (Accuracy is higher with lower RMSE and higher $\mathrm{R}^{2}$ values).

Overall, the three versions of the QMC method are the most efficient sampling techniques representing the reference data, followed by the importance sampling technique. In contrast, the MCMC and MC provide the worst representation for the reference dataset with fewer samples' generation, respectively.

\subsection{System Loadability Analysis}

In order to confirm the most efficient and accurate sampling technique generation, as discussed above, to use in the probabilistic voltage stability analysis, the continuation power flow has been performed in DIgSILENT PowerFactory software for the reference dataset (8760 samples) and the sample datasets with different sizes that are generated by applying the six sampling techniques. Figure 9 shows the PDF of the system loadability for $150,300,1000$, and 8760 simulations by applying the six sampling techniques compared to the reference data. Moreover, the RMSE and $\mathrm{R}^{2}$ values of the system loadability for a different number of simulations compared to the reference dataset are shown in Figure 10.

It can be seen from Figure 9 that the accuracy of the sampling techniques is improved by increasing the number of simulations. By performing the continuation power flow for a large number of wind speed datasets ( 8760 samples), it can be noticed that all the sampling techniques follow the obtained result of the reference dataset accurately, as presented in Figure 9d. This observation has also been verified by low RMSE and high $\mathrm{R}^{2}$ values for the six sampling techniques, as shown in Figure 10, which is consistent with the previous result presented in Figures 7 and 8.

On the other hand, to identify and validate the most efficient sampling techniques (as mentioned above in Section 4.1), the continuation power flow has been performed for fewer random sample generations (150 and 300 samples). It can be seen from Figure 9a,b that the three versions of the QMC method provide the closest and the most acceptable results that follow the reference data in comparison to other techniques. Conversely, the MCMC and MC present the most unsatisfactory representation for the reference dataset, respectively. This result can be verified from Figure 10, which shows that the three versions of the QMC method have the lowest RMSE values and the highest $R^{2}$ values in contrast to other sampling techniques for the 150 and 300 random samples. This result is further consistent with the RMSE and $\mathrm{R}^{2}$ values that are shown in Figure 8. 


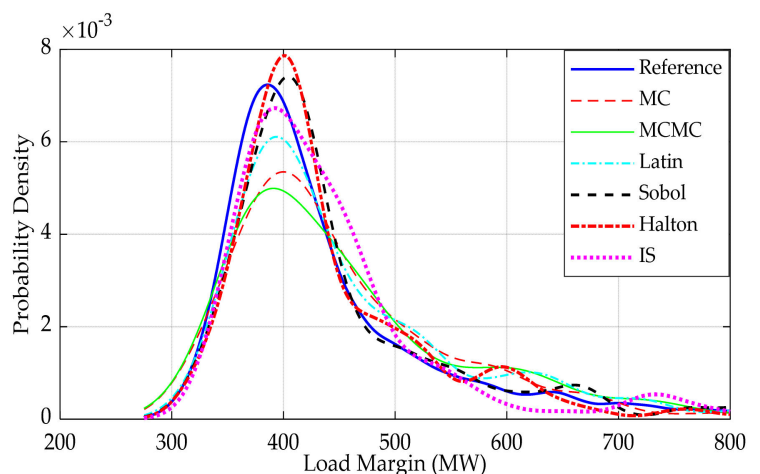

(a)

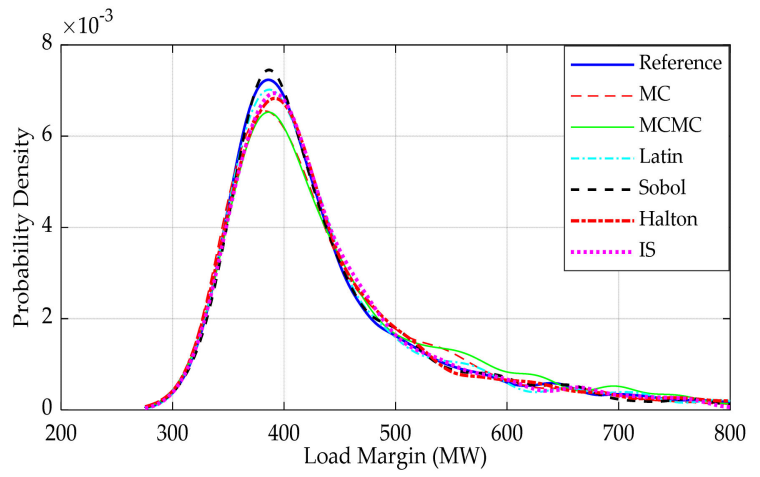

(c)

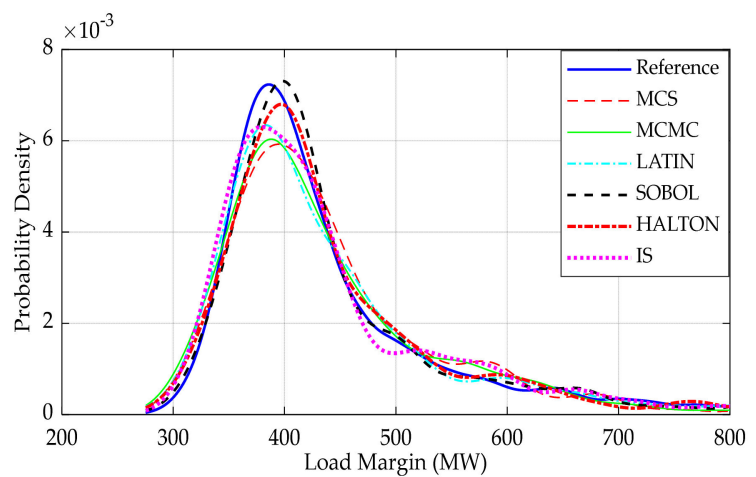

(b)

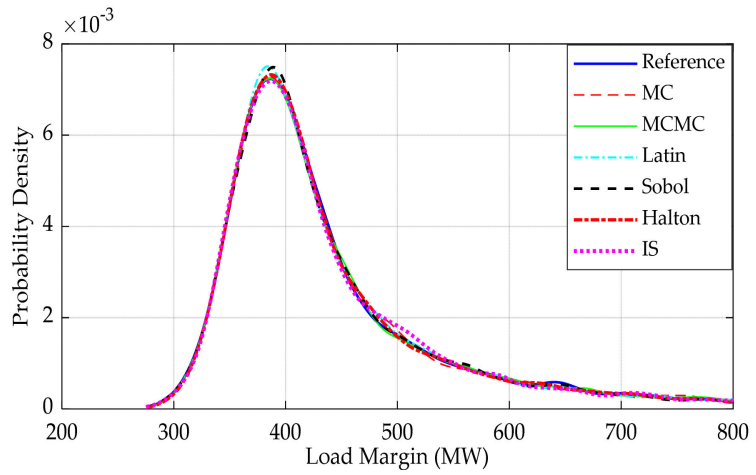

(d)

Figure 9. The PDF of the system loadability by using the six sampling techniques compared to the reference data: (a) 150 simulations; (b) 300 simulations; (c) 1000 simulations; (d) 8760 simulations (cover the number of hours of the year).

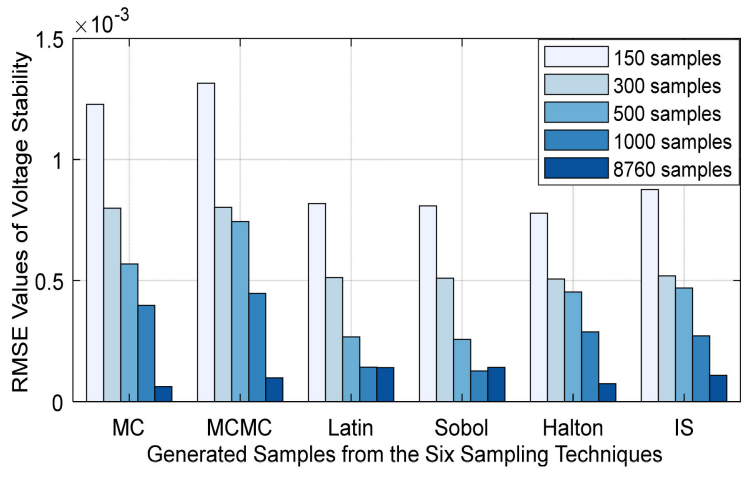

(a)

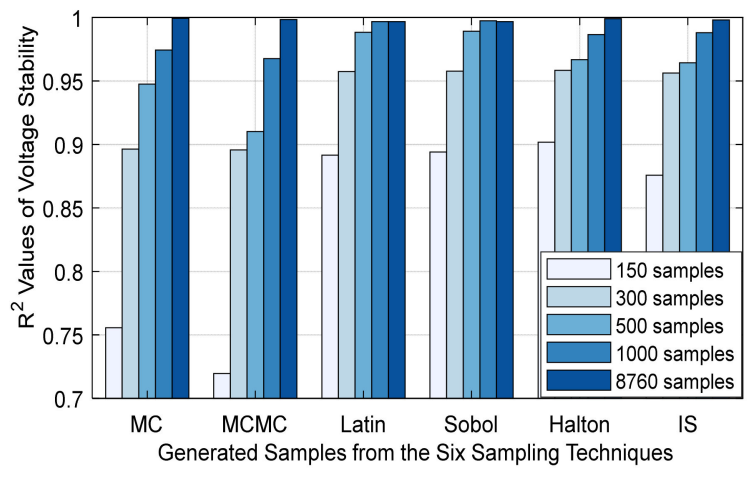

(b)

Figure 10. The (a) RMSE and (b) $\mathrm{R}^{2}$ values of 150, 300, 500, 1000, and 8760 simulations of the system loadability with the six sampling techniques compared to the reference dataset.

Overall, all of these sampling techniques can be employed to analyze the probabilistic voltage stability assessment; however, some of them, such as MC and MCMC, cannot be applied in a realistic large-scale power system since they require a large number of simulations to provide an accurate and acceptable result. On the other hand, the three versions of the QMC method are efficient and accurate, and therefore, they can be utilized for a realistic and large-scale power system.

Figure 11 presents the $\mathrm{R}^{2}$ values of the generated wind speed samples by using the six sampling techniques and the system loadability based on the generated datasets against the number of the samples. This figure clearly shows the accuracy vs. efficiency of each sampling technique and a comparison among them, in order to identify the suitability of each method that can be employed to generate wind data samples and perform power system voltage stability studies. 


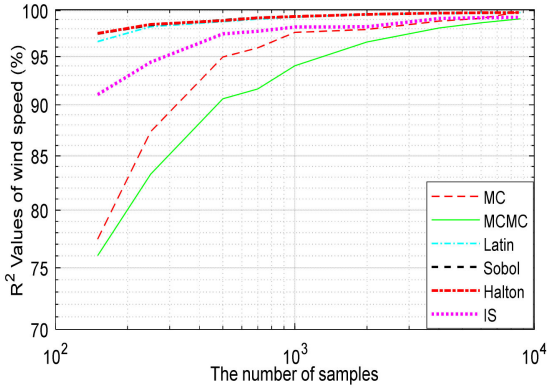

(a)

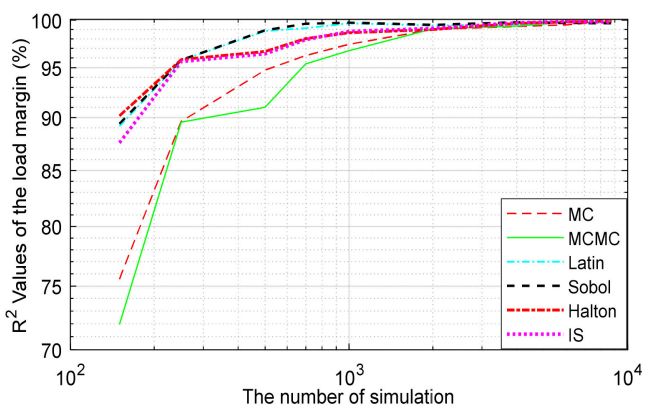

(b)

Figure 11. The $R^{2}$ values vs. the number of the samples for (a) the generated wind speed, and (b) the system loadability by using the six sampling techniques.

It can be seen from Figure 11a that the accuracy of generating random wind speed data is about $76 \%, 77 \%, 91 \%$, and $96 \%$ for MCMC, MC, IS, and QMC techniques, respectively, for 150 samples. The accuracy increases for all methods with the increase in the number of simulations and reaches $90 \%, 95 \%, 97 \%$, and $99 \%$ for MCMC, MC, IS, and QMC techniques, respectively, for 500 samples. The accuracy of all methods becomes almost saturated above 3000 simulations and is shown to be $97.5 \%, 98.5 \%, 98.5 \%$, and $99.5 \%$ for MCMC, MC, IS, and QMC techniques, respectively. The same trend is also evident from Figure 11b.

Moreover, it can be observed from Figure 11 that if the number of samples is too low $(<500)$, then the accuracy of the methods varies, and some results are not acceptable. On the other hand, if the number of samples is high (>1500), the performances of the methods are good, but the higher number of simulations could be a barrier for applying these methods to large-scale networks.

From these results, it can be concluded that the three versions of the QMC method, which include Sobol, Halton, and Latin hypercube, and the importance sampling technique are the most efficient sampling techniques, respectively. In contrast, the MCMC method (followed by the MC) provides the lowest efficient sample datasets for representing wind speed data. This conclusion is expected, as the QMC methods produce random samples with low-discrepancy sequences, which are based on uniformly distributed sequences.

Overall, the dataset generated by applying the QMC method can lead to an accurate and acceptable result with fewer generated samples than other sampling techniques. On the other hand, the $\mathrm{MC}$ and the MCMC require a large number of simulations to provide accurate and satisfactory results, which need more than 2000 simulations to obtain $97 \%$ accuracy, as can be seen from Figure 11. This requirement limits the applicability of these methods and renders them almost impossible to be employed for a large-scale power system.

\section{Conclusions}

The paper presented a comparative analysis to identify efficient and accurate sampling techniques, to evaluate their appropriateness for probabilistic voltage stability analysis in large-scale power systems since the traditional MC method is time-consuming and computationally expensive. Accordingly, various sampling techniques were implemented, including MC, three versions of QMC (Sobol, Halton, and Latin Hypercube), MCMC, and importance sampling techniques. The continuation power flow was performed in the IEEE-39 bus network to validate the suitability of the most efficient and accurate sampling techniques for probabilistic voltage stability analysis based on the RMSE and $\mathrm{R}^{2}$ criteria.

The obtained simulation results indicate that the accuracy of the six sampling techniques is increased by increasing the number of random samples based on the wind speed generation analysis. Additionally, by producing a large number of random samples, the six sampling techniques perfectly followed the reference data. This has also been shown by the voltage stability simulation. 
In terms of efficiency, on the other hand, the obtained results show that the three versions of the QMC are the most efficient and accurate sampling techniques that provide a better representation by producing fewer random number samples for the reference data. In contrast, the MCMC (followed by $\mathrm{MC}$ ) provides the worst generated sample datasets to represent the reference data with fewer samples based on wind speed analysis, which is further confirmed by the system loadability analysis.

This research enables more informed determinations to be made when choosing an efficient sampling technique for probabilistic voltage stability analysis in large-scale uncertain power systems, and especially for performing high-resolution dynamic studies. Additionally, the three versions of the QMC could be utilized in other aspects of power system stability, security, and reliability prevention studies in large power system networks. Finally, the development and implementation of novel efficient sampling techniques is an ongoing area of research concerning their appropriateness for application in probabilistic voltage stability analysis.

Author Contributions: Conceptualization, methodology, simulation, writing-original draft preparation by M.A.; conceptualization, methodology, writing - review and editing, supervision by K.N.H.; conceptualization, writing — review and editing, supervision by L.M.; simulation, writing-review and editing by M.T.R. All authors have read and agreed to the published version of the manuscript.

Funding: There is no funding to be reported for this publication.

Conflicts of Interest: The authors declare no conflict of interest.

\section{References}

1. Kenari, M.T.; Sepasian, M.S.; Nazar, M.S. Probabilistic voltage stability assessment of distribution networks with wind generation using combined cumulants and maximum entropy method. Int. J. Electr. Power Energy Syst. 2018, 95, 96-107. [CrossRef]

2. Qi, B.; Hasan, K.N.; Milanovic, J.V. Identification of critical parameters affecting voltage and angular stability considering load-renewable generation correlations. IEEE Trans. Power Syst. 2019, 34, 2859-2869. [CrossRef]

3. Xiang, M.; Yu, J.; Yang, Z.; Yang, Y.; Yu, H.; He, H. Probabilistic power flow with topology changes based on deep neural network. Int. J. Electr. Power Energy Syst. 2020, 117, 105650. [CrossRef]

4. Vittal, E.; O'Malley, M.; Keane, A. A steady-state voltage stability analysis of power systems with high penetrations of wind. IEEE Trans. Power Syst. 2010, 25, 433-442. [CrossRef]

5. Meegahapola, L.; Littler, T. Characterisation of large disturbance rotor angle and voltage stability in interconnected power networks with distributed wind generation. IET Renew. Power Gener. 2015, 9, 272-283. [CrossRef]

6. Adetokun, B.B.; Muriithi, C.M.; Ojo, J.O. Voltage stability assessment and enhancement of power grid with increasing wind energy penetration. Int. J. Electr. Power Energy Syst. 2020, 120, 105988. [CrossRef]

7. Hasan, K.N.; Preece, R.; Milanović, J.V. Existing approaches and trends in uncertainty modelling and probabilistic stability analysis of power systems with renewable generation. Renew. Sustain. Energy Rev. 2019, 101, 168-180. [CrossRef]

8. Milanovic, J.V. Probabilistic stability analysis: The way forward for stability analysis of sustainable power systems. Philos. Trans. A Math. Phys. Eng. Sci. 2017, 375. [CrossRef]

9. Zhang, J.; Tse, C.; Wang, K.; Chung, C. Voltage stability analysis considering the uncertainties of dynamic load parameters. IET Gener. Transm. Distrib. 2009, 3, 941-948. [CrossRef]

10. Almeida, A.B.; De Lorenci, E.V.; Leme, R.C.; De Souza, A.C.Z.; Lopes, B.I.L.; Lo, K. Probabilistic voltage stability assessment considering renewable sources with the help of the pv and qv curves. IET Renew. Power Gener. 2013, 7, 521-530. [CrossRef]

11. Muñoz, J.; Cañizares, C.; Bhattacharya, K.; Vaccaro, A. An affine arithmetic-based method for voltage stability assessment of power systems with intermittent generation sources. IEEE Trans. Power Syst. 2013, 28, 4475-4487. [CrossRef]

12. Rodrigues, A.B.; Prada, R.B.; da Silva, M.D.G. Voltage stability probabilistic assessment in composite systems: Modeling unsolvability and controllability loss. IEEE Trans. Power Syst. 2010, 25, 1575-1588. [CrossRef]

13. Deng, W.; Zhang, B.; Ding, H.; Li, H. Risk-based probabilistic voltage stability assessment in uncertain power system. Energies 2017, 10, 180. [CrossRef]

14. Tang, F.; Zhou, S.; Zhang, Q.; Liao, Q.; Liu, D.; Yin, Q. A static voltage stability assessment scheme of power systems considering charging state of electric vehicles and load fluctuation limits. In Proceedings of the 2017 IEEE Power \& Energy Society General Meeting, Chicago, IL, USA, 16-20 July 2017; pp. 1-5.

15. Zhang, J.; Tse, C.; Wang, W.; Chung, C. Voltage stability analysis based on probabilistic power flow and maximum entropy. IET Gener. Transm. Distrib. 2010, 4, 530-537. [CrossRef]

16. Preece, R.; Woolley, N.C.; Milanovic, J.V. The probabilistic collocation method for power-system damping and voltage collapse studies in the presence of uncertainties. IEEE Trans. Power Syst. 2013, 3, 2253-2262. [CrossRef] 
17. Zheng, C.; Kezunovic, M. Impact of wind generation uncertainty on power system small disturbance voltage stability: A pcm-based approach. Electr. Power Syst. Res. 2012, 84, 10-19. [CrossRef]

18. Schellenberg, A.; Rosehart, W.; Aguado, J.A. Cumulant-based stochastic nonlinear programming for variance constrained voltage stability analysis of power systems. IEEE Trans. Power Syst. 2006, 21, 579-585. [CrossRef]

19. Preece, R.; Huang, K.; Milanovic, J.V. Probabilistic small-disturbance stability assessment of uncertain power systems using efficient estimation methods. IEEE Trans. Power Syst. 2014, 29, 2509-2517. [CrossRef]

20. Wangdee, W.; Billinton, R. Bulk electric system well-being analysis using sequential monte carlo simulation. IEEE Trans. Power Syst. 2006, 21, 188-193. [CrossRef]

21. Preece, R.; Milanovic, J.V. Efficient estimation of the probability of small-disturbance instability of large uncertain power systems. IEEE Trans. Power Syst. 2016, 31, 1063-1072. [CrossRef]

22. Hasan, K.N.; Preece, R.; Milanovic, J.V. Priority ranking of critical uncertainties affecting small-disturbance stability using sensitivity analysis techniques. IEEE Trans. Power Syst. 2017, 32, 2629-2639. [CrossRef]

23. Kundur, P.; Paserba, J.; Ajjarapu, V.; Andersson, G.; Bose, A.; Canizares, C.; Hatziargyriou, N.; Hill, D.; Stankovic, A.; Taylor, C. Definition and classification of power system stability ieee/cigre joint task force on stability terms and definitions. IEEE Trans. Power Syst. 2004, 19, 1387-1401.

24. Meegahapola, L.; Sguarezi, A.; Bryant, J.S.; Gu, M.; Conde D, E.R.; Cunha, R. Power system stability with power-electronic converter interfaced renewable power generation: Present issues and future trends. Energies 2020, 13, 3441. [CrossRef]

25. Eremia, M.; Bulac, C. Voltage stability. In Handbook of Electrical Power System Dynamics: Modeling, Stability, and Control; Wiley: New York, NY, USA, 2013; pp. 657-736.

26. Kundur, P.; Balu, N.J.; Lauby, M.G. Power System Stability and Control; McGraw-Hill: New York, NY, USA, 1994 ; Volume 7.

27. Xu, X.; Yan, Z.; Shahidehpour, M.; Wang, H.; Chen, S. Power system voltage stability evaluation considering renewable energy with correlated variabilities. IEEE Trans. Power Syst. 2017, 33, 3236-3245. [CrossRef]

28. Martinez, J.A.; Guerra, G. A parallel monte carlo method for optimum allocation of distributed generation. IEEE Trans. Power Syst. 2014, 29, 2926-2933. [CrossRef]

29. El-Khattam, W.; Hegazy, Y.; Salama, M. Investigating distributed generation systems performance using monte carlo simulation. IEEE Trans. Power Syst. 2006, 21, 524-532. [CrossRef]

30. Gevorgian, V.; Zhang, Y.; Ela, E. Investigating the impacts of wind generation participation in interconnection frequency response. IEEE Trans. Sustain. Energy 2015, 3, 1004-1012. [CrossRef]

31. Ruttledge, L.; Miller, N.W.; O'Sullivan, J.; Flynn, D. Frequency response of power systems with variable speed wind turbines. IEEE Trans. Sustain. Energy 2012, 3, 683-691. [CrossRef]

32. Papadopoulos, P.N.; Milanović, J.V. Probabilistic framework for transient stability assessment of power systems with high penetration of renewable generation. IEEE Trans. Power Syst. 2017, 32, 3078-3088. [CrossRef]

33. Shi, L.; Sun, S.; Yao, L.; Ni, Y.; Bazargan, M. Effects of wind generation intermittency and volatility on power system transient stability. IET Renew. Power Gener. 2014, 8, 509. [CrossRef]

34. Hasan, K.N.; Preece, R.; Milanovic, J.V. The influence of load on risk-based small-disturbance security profile of a power system. IEEE Trans. Power Syst. 2018, 33, 557-566. [CrossRef]

35. Huang, H.; Chung, C.; Chan, K.W.; Chen, H. Quasi-monte carlo based probabilistic small signal stability analysis for power systems with plug-in electric vehicle and wind power integration. IEEE Trans. Power Syst. 2013, 28, 3335-3343. [CrossRef]

36. Zou, B.; Xiao, Q. Solving probabilistic optimal power flow problem using quasi monte carlo method and ninth-order polynomial normal transformation. IEEE Trans. Power Syst. 2014, 1, 300-306. [CrossRef]

37. Collin, A.J.; Tsagarakis, G.; Kiprakis, A.E.; McLaughlin, S. Development of low-voltage load models for the residential load sector. IEEE Trans. Power Syst. 2014, 29, 2180-2188. [CrossRef]

38. Neal, R.M. Slice sampling. In Annals of Statistics; JSTOR: New York, NY, USA, 2003; pp. 705-741.

39. Souxes, T.; Granitsas, I.-M.; Vournas, C. Effect of stochasticity on voltage stability support provided by wind farms: Application to the hellenic interconnected system. Electr. Power Syst. Res. 2019, 170, 48-56. [CrossRef]

40. Fan, Y.; Zai, X.; Qian, H.; Yang, X.; Liu, L.; Zhu, Y. Transient stability analysis of power system based on bayesian networks and main electrical wiring. In Proceedings of the 2009 Asia-Pacific Power and Energy Engineering Conference, Wuhan, China, 27-31 March 2009; pp. 1-4.

41. Metelli, A.M.; Papini, M.; Montali, N.; Restelli, M. Importance sampling techniques for policy optimization. J. Mach. Learn. Res. 2020, 21, 1-75.

42. Wang, Y.; Guo, C.; Wu, Q.; Dong, S. Distribution. Adaptive sequential importance sampling technique for short-term composite power system adequacy evaluation. IET Gener. Transm. Distrib. 2014, 4, 730-741. [CrossRef]

43. Cai, J.; Xu, Q.; Cao, M.; Yang, B. A novel importance sampling method of power system reliability assessment considering multi-state units and correlation between wind speed and load. Int. J. Electr. Power Energy Syst. 2019, 109, 217-226. [CrossRef]

44. Tinitana, J.C.C.; Correa-Florez, C.A.; Patino, D.; Vuelvas, J. Spatio-temporal kriging based economic dispatch problem including wind uncertainty. Energies 2020, 13, 6419. [CrossRef]

45. Memon, Z.A.; Trinchero, R.; Manfredi, P.; Canavero, F.; Stievano, I.S. Compressed machine learning models for the uncertainty quantification of power distribution networks. Energies 2020, 13, 4881. [CrossRef] 
46. Ding, S.; Pichon, L. Sensitivity analysis of an implanted antenna within surrounding biological environment. Energies 2020, 13, 996. [CrossRef]

47. Khodayar, M.; Wang, J. Spatio-temporal graph deep neural network for short-term wind speed forecasting. IEEE Trans. Sustain. Energy 2018, 10, 670-681. [CrossRef]

48. Filik, T. Improved spatio-temporal linear models for very short-term wind speed forecasting. Energies 2016, 9, 168. [CrossRef]

49. Lorca, A.; Sun, X.A. Adaptive robust optimization with dynamic uncertainty sets for multi-period economic dispatch under significant wind. IEEE Trans. Power Syst. 2014, 30, 1702-1713. [CrossRef]

50. Qian, H.; Hongkun, C.; Yubo, T.; Ruixi, Y.; Ling, W.; Pan, H. Wind speed spatio-temporal forecasting of wind farms based on universal kriging and Bayesian dynamic model. In Proceedings of the 2014 International Conference on Power System Technology, Chengdu, China, 20-22 October 2014; pp. 2897-2902.

51. Akdağ, S.A.; Dinler, A. A new method to estimate weibull parameters for wind energy applications. Energy Convers. Manag. 2009, 50, 1761-1766. [CrossRef]

52. Pai, M. Energy Function Analysis for Power System Stability; Springer Science \& Business Media: Berlin/Heidelberg, Germany, 2012.

53. Met Office. Met Office Midas Open: UK Land Surface Stations Data (1853-Current). Centre for Environmental Data Analysis, Date of Citation. 2019. Available online: http:/ / catalogue.Ceda.Ac.Uk/uuid/dbd451271eb04662beade68da43546e1 (accessed on 20 October 2020).

54. Negnevitsky, M.; Nguyen, D.H.; Piekutowski, M. Risk assessment for power system operation planning with high wind power penetration. IEEE Trans. Power Syst. 2015, 30, 1359-1368. [CrossRef] 